\title{
Stochastic operation of isolated microgrids: aggregating-rule- based optimization versus standard approaches
}

\author{
Davide Fioriti ${ }^{1, *}$, Giovanni Lutzemberger ${ }^{1}$, Davide Poli $^{1}$ and Pablo Duenas-Martinez ${ }^{2}$ \\ ${ }^{1}$ Department of Energy, Systems, Territory and Construction Engineering, University of Pisa, 56122 Pisa, Italy \\ ${ }^{2}$ MIT Energy Initiative, Massachusetts Institute of Technology, 02139 Cambridge (MA), United States
}

\begin{abstract}
Stochastic operation of power systems has risen attention of researchers as fluctuating energy sources like renewables are being increasingly integrated into existing grids. Uncertainties can be higher in small power systems like isolated microgrids, where both renewables and load can be extremely unpredictable, thus causing increasing operating costs and business risks. In the last years, many approaches have been proposed to account for uncertainties in off-grid microgrids, usually simulating several size, load and renewables scenarios. Among them, a simplified stochastic approach, namely Aggregating-Rule-based Stochastic Optimization (ARSO), which decomposes the N-scenario problem into $\mathrm{N}$ deterministic subproblems whose solutions are finally processed and aggregated, has been recently proposed with interesting results in terms of optimality of results and computational requirements. In this paper, two ARSO approaches are compared with standard stochastic and deterministic methodologies used to operate isolated microgrids, to assess advantages and drawbacks of all these techniques and their ability in handling uncertainties. The two ARSO methodologies differ in the aggregating rule: to take into account the load and RES forecasting errors, the Improved-ARSO employs a Monte Carlo procedure, whereas the Mixed ARSO technique makes use of statistical rules. A numerical case study for a typical isolated microgrid in Africa is proposed and discussed.
\end{abstract}

Keywords: Aggregating-Rule-based Stochastic Optimization (ARSO); scenario decomposition; mini-grid; hybrid energy system; Monte Carlo scenarios

\section{Introduction}

Isolated microgrids are well known to be an efficient solution to supply electricity by exploiting local sources, without the burden of installing long and expensive power lines. The use of these systems is very common in islands, but, in the last years, has been increasingly used also in rural areas of developing countries, to support rural electrification [1].

Representing the backbone of over $97 \%$ of the installed off-grid systems [1], diesel generators have been widely used as a very cheap, easy to operate and fast installation technology; however, their operating costs in remote areas are very high, and fluctuate with the fuel price. Nowadays, hybrid systems that couple multiple sources and energy storage devices are becoming the standard, with added advantage of coping with environmental concerns [2]. Even if the recent decreasing in the installation cost (CAPEX) makes renewable sources cheap solutions on a long-term basis, they are subject to seasonality and fluctuations of the power source, thus affecting the operating costs of the fuel-fired backup generators, as well as the reliability and the quality of service. For these reasons, renewables and load uncertainties must be addressed in both the design and the operational phase to hedge business risks.

Traditional hybrid microgrids are usually operated under priority-list rules [3] that dispatch first the renewable sources, then the electrical storages and finally the fuel-fired generators, expected to be used as a simple backup source. Based on "if-then" rules, approaches like Load Following and Cycle Charging are very simple, robust, and extremely easy to be implemented in the majority of control devices.

Recently, predictive approaches based on the periodic forecasting of renewables and load have shown promising results, with operational savings around 6$10 \%$ with respect to priority-list strategies, thus captivating the attention of many private companies [4], [5]. Based on the expected power profiles of demand and renewable energy production of the following hours, these approaches deterministically calculate the

\footnotetext{
*Corresponding author: davide.fioriti@ing.unipi.it
} 
dispatch of the system that minimizes the operating costs of service, usually including the expenses for the fuel, the maintenance and the unserved load (a measurement of service quality). These predictive approaches, hereinafter referred to as Deterministic Optimization (DO), rely on optimization techniques that are more complex than priority-list methods and require good forecasts, although savings have revealed consistent. State-of-art formulations are based on Mixed-Integer Linear Programming (MILP), which has proven to be robust and guarantee reaching the global optimum [6]. Furthermore, MILP computational requirements are usually consistent with rolling-horizon approaches, which can also be implemented in commercial devices [4], [5]. Nevertheless, in their basic deterministic formulation (i.e. one single forecasted power profile), none of these techniques consider uncertainties in the optimization phase. Unavoidable forecasting errors thus lead to possible sub-optimal solutions in real operation.

Stochastic techniques have proven to be promising as they can reduce the operating system costs and the amount of unserved energy with respect to traditional priority rules [6]-[8]. Uncertainties are usually modelled by several possible scenarios of load and renewable energy production, which together approximate the probability density function of the RES/load forecasting errors. The generated profiles are then used by an optimization algorithm aimed to identify the best dispatching of resources, which in standard Stochastic Optimization (SO) minimizes the expected operating costs by a weighted-average of the cost of each scenario, obtaining each weight from each scenario probability according to the Sample Average Approximation (SAA) method [9]. Several studies confirmed the profitability of SO for both large and small power systems [6], [10], [11]. Authors in [6] highlighted that SO performed better among several techniques for a large power system with high penetration of renewable production, while in [10], [11], SO was discussed for isolated systems, turning out to be up to $35 \%$ cheaper than the DO solution.

However, the computational requirements of conventional SO procedures are consistently higher than DO. In fact, the computational time increases more than linearly with the number of scenarios, especially when several binary variables are involved; therefore, a tradeoff between optimality of results and time is required [12]. For this reason, several decomposition techniques are usually adopted to simplify stochastic approaches, like Benders or Danzig-Wolfe decompositions, often based on scenarios [13]. On the other hand, scenario reduction methods are used to reduce the number of scenarios to consider, while preserving the stochastic characteristics of the distribution, thus reducing computational time [14].

To overcome complexity and computational requirements of standard SO procedures, other approaches, for the first time referred to as AggregatingRule-based Stochastic Optimization (ARSO) in [15], decompose the $\mathrm{N}$-scenarios problem into $\mathrm{N}$ deterministic sub-problems and then aggregate the results based on different methodologies [15]-[18].
While the deterministic optimization of each subproblem is similar, the difference between the different ARSO approaches is mainly related to the aggregation criterion, which can be statistical-based [16], weighted-average [18], cost-based [15], or mixed [17]. Authors in [16] agglomerate the solution from the first stage to identify the probabilistic dispatching of the system by aggregating the results. A mixed ARSO methodology was proposed in [17] for a reconfigurable microgrid: the grid topology was set with a statistical approach, while the generator scheduling with a weighted-average approach. Instead, the method proposed in [15] collects the optimal scheduling of the deterministic sub-problems and simulates the corresponding real-time system operation of each of them to evaluate the expected operating costs, given the probability density function of the forecasting errors. This methodology has performed very well with respect to traditional Stochastic Optimization (SO), but limited comparisons to ARSO approaches have been developed.

The methodology suggested in [15] has shown promising results, but further studies are needed to highlight benefits. Therefore, in this paper we propose a comparison between mixed and cost-based ARSO methodologies and typical SO and DO approaches. A numerical case study related to an off-grid system in Uganda is shown and discussed. Additionally, a sensitivity analysis regarding the initial state of the system is carried out, to evaluate how the various approaches perform under different starting conditions.

\section{The system}

\subsection{Topology}

Being strongly affected by the forecasting errors of the load and renewable sources, the mini-grid under consideration is a typical off-grid system installed in developing countries, composed by a photovoltaic plant, an inverter, a battery storage, a battery converter and a diesel generator, used as a backup source. The typical $\mathrm{AC}$ configuration where all sources are coupled at the AC busbar has been chosen, as it is modular and suitable for rural applications [19]. The photovoltaic system and batteries are coupled at the AC busbar by means of converters, while the fuel-fired generator is directly tied.

\subsection{The operating strategy}

The system is operated using the following strategy: the day-ahead scheduling of the dispatchable resources (fuel-fired generators and storages) is firstly optimized for the following 24 hours, then during real-time, the operation is adjusted to cope with unavoidable forecasting errors. Priority-list rules are used for realtime balancing: first renewable sources are dispatched to correct deviations with respect to the expected operation, then storage, and finally the fuel-fired generators, similarly to [15], [20].

It is worth noticing that other papers have proposed DO methodologies for the optimal scheduling of these systems [20]; however, few studies compared the use of 
different operating strategies in the scheduling level. Here, we propose a comparison in the day-ahead dispatching algorithm; in particular, the typical DO method is compared to SO and to two different ARSO strategies. The case study was developed to be comparable to results reported in [15]. As SO and DO techniques are well known, in the following we focus on the description of ARSO methodologies, while the mathematical problem of DO and SO is based on [15].

\section{The optimization strategy}

\subsection{Description}

This section details the optimization procedures used in the proposed paper. The structure of the conventional SO and ARSO problems are shown in Fig. 1 and Fig. 2, respectively. The typical SO formulation calculates the scheduling that minimizes the expected operating system costs, evaluated with SAA method [9], using a number of scenarios that capture forecasting uncertainties. In this formulation, the optimization is performed using all scenarios together, hence the computational costs sharply increase with the number of scenarios. Conversely, in ARSO methodologies, each scenario is optimized independently (first stage) and later an aggregation algorithm (second stage) identifies the final dispatch according to a rule that is usually statistical, weighted-average, cost-based or mixed. In all formulations, the expenses for the fuel, the generator's maintenance, the load shedding and the battery overuse are considered. This last term takes into account that overusing the battery in a time-horizon wears the reserve for the following hours, which would increase the costs in the following time-step. To prevent this, the batteryoveruse term is non-zero only when the energy stored in the battery at the end of the optimization period falls below a fixed level, set to the initial storage level.

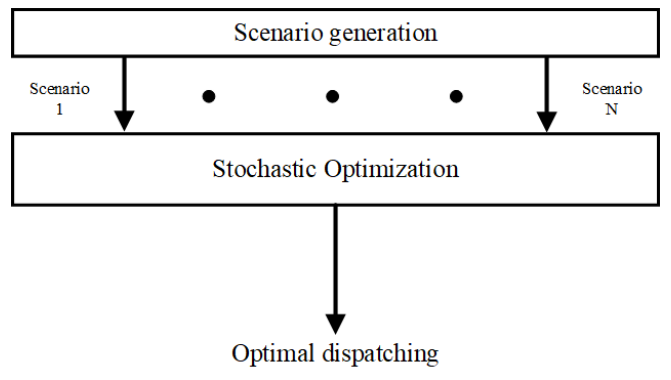

Fig. 1. Stochastic Optimization.

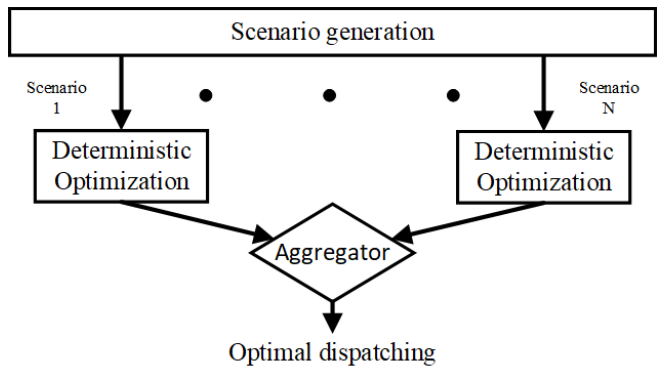

Fig. 2. Aggregating-Rule-based Stochastic Optimization (ARSO).
In this paper, a statistical (M-ARSO) and a novel cost-based (I-ARSO) ARSO methodologies are described and applied, as well as both the standard SO and DO approaches.

\subsection{Mixed-rule ARSO (M-ARSO)}

Typical ARSO approaches identify the optimal dispatching by analyzing the scheduling achieved by the deterministic optimizations carried out in the first stage; the final dispatching is selected according to statistical rules (i.e. the most recurring scheduling) or weightedaverage approaches. In particular, on/off decisions like the status of generators ("Unit Commitment") are often selected by using statistical approaches, while the power level requested to storage and committed generating units ("power dispatching") is usually selected using a weighted-average approach [15].

In this paper, we consider a mixed-rule aggregator based on the following algorithm:

1. The optimal dispatching corresponding to each one of the N RES/load scenarios is found using a deterministic optimization routine, later described.

2. The most recurring unit commitment among the results of $\mathrm{N}$ scenarios is identified and the start-up and shut-down of fuel-fired generators is scheduled accordingly.

3. The average value of power profiles corresponding to the selected unit commitment is calculated and assigned to generators and storage devices.

\subsection{Improved ARSO (I-ARSO)}

Firstly proposed in [15], the Improved ARSO (IARSO) model developed by the authors is the two-stage methodology depicted in Fig. 3 In the first stage, each one of the $\mathrm{N}$ RES/load scenarios is optimized independently using the DO algorithm. In the second stage, each one of the $\mathrm{N}$ optimal dispatching strategies assessed in the first stage is simulated for M Monte Carlo scenarios allowing to obtain the average expected real-time costs of each possible dispatching strategy of the first stage. The aggregator selects the dispatching strategy with the lowest expected operating cost, averaged on the $\mathrm{M}$ corresponding Monte Carlo

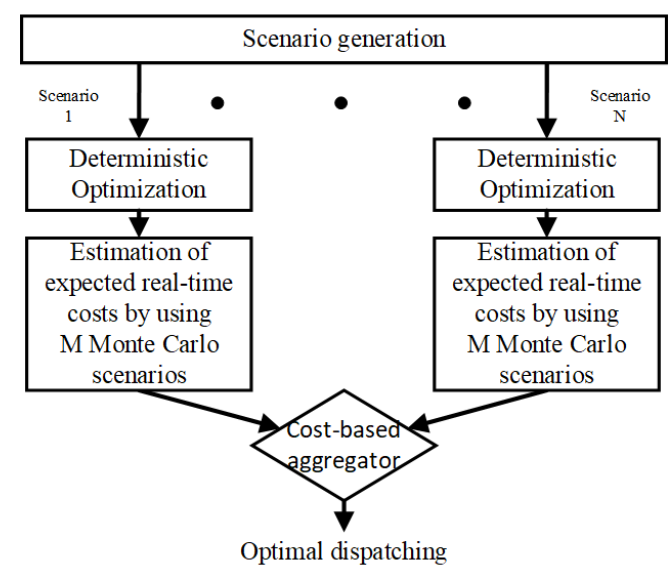

Fig. 3. The novel I-ARSO technique. 
simulations [15]. It is worth noticing that the parameters of this algorithm are $\mathrm{N}$ and $\mathrm{M}$, i.e. the number of scenarios to be used in the first and second stage of the method, respectively.

\subsection{Standard Stochastic Optimization (SO)}

The two-stage SO approach developed in this activity identifies the scheduling of the resources of the microgrid that minimizes the expected operating costs of the system; the uncertainties are modelled by means of scenarios using the SAA algorithm [9]. While the first-stage variables are the commitment and dispatching of the generator, the second-stage variables model the fast rules based on priority-list rules, used to keep the system stable. The model described in [15] was taken as reference and implemented in this activity.

\subsection{Standard Deterministic Optimization (DO)}

The standard DO formulation aims at scheduling generators and storages to minimize the operating costs of the system, considering a single forecast of the demand and the renewable energy production. The corresponding mathematical formulation is similar to the one of SO, but for a single scenario. This formulation is also used to optimize the first-stage scenarios of MARSO and I-ARSO methodologies; the mathematical details of the approach can be found in [15].

\section{Case Study}

\subsection{Description}

The proposed case study is developed for a possible mini-grid serving 100 households in Soroti, Uganda; in particular, we investigated the day-ahead dispatching of a typical day during the rainy season, which is the most critical period because the renewable production decreases. The load profile and the renewable energy production were estimated using the same methodology described in [15], so that the results can be comparable. The system under consideration is composed by a 140kWp PV system, a 250-kWh lithium battery, a 70-kW inverter and a $30-\mathrm{kW}$ fuel-fired generator [15].

\subsection{Sensitivity analysis and comparison methodology}

In this study, we want to evaluate the effects of different predictive approaches to be used in the day-ahead scheduling phase (I-ARSO, M-ARSO, SO and DO) into the operating cost of a typical day of the rainy season, as described in Section 2.2. Due to its importance, we include a sensitivity analysis with respect to the initial storage level.

In order to compare the different approaches on a common ground, first each approach is applied to calculate the corresponding optimal dispatching, then the real-time system operation of that scheduling is simulated for a large number of scenarios (100000), to evaluate the expected equivalent costs, similarly to [21].

\subsection{Other parameters}

The number of scenarios of the first- and secondstage of I-ARSO approaches was set to $\mathrm{N}=250$ and $M=1000$, respectively. Instead, for the SO approach, in order to reduce the computational requirements without affecting the stochastic properties of simulations, a scenario reduction technique was used to reduce a large number of Monte Carlo scenarios (1000) to a lower number (6) by using a k-means algorithm, as in [15].

The fuel price was set to $1 \$ / 1$ and the economic value of the energy not served was assumed equal to $2 \$ / \mathrm{kWh}$. The maintenance fee of the generator is $0.6 \$ / \mathrm{kWh}$ and the efficiency of batteries is $88 \%$ (round-trip). Finally, the equivalent cost of discharging the battery more than the initial level was assumed equal to $0.33 \$ / \mathrm{h}$, which is equivalent to the specific generator consumption at the rated power including maintenance.

\section{Results}

The results of the day-ahead system scheduling for the proposed case study are shown in Tab. 3 and Fig. 4, including the sensitivity analysis with respect to the initial battery level $(20 \%, 40 \%$ and $60 \%)$ and using different operating strategies (I-ARSO, M-ARSO, DO and SO). Fig. 4 reports the outcomes of the two ARSO methodologies, to highlight the effects of both M-ARSO and I-ARSO on the unit commitment of the fuel-fired generator. In particular, Fig. $4 \mathrm{a}$, Fig. $4 \mathrm{~b}$ and Fig. $4 \mathrm{c}$ report all the different commitment sequences resulted by the $\mathrm{N}$ first-stage sub-problems: white and black dots highlight hours when the generator is on or off, respectively. It is worth noticing that the number $\mathrm{K}$ of different commitment sequences is lower than $\mathrm{N}$ (250), because the same unit commitment sequence can be identified as optimal in more than one load/RES firststage scenario. The occurrence probability of each configuration, as used in the M-ARSO procedure, is depicted in Fig. 4d, Fig. 4e and Fig. 4f. In the second stage of the I-ARSO technique, all $\mathrm{N}$ optimal power dispatching profiles obtained in the first stage were simulated to observe what would happen in real time, by using the Monte Carlo procedure described in Section 3.3. For the sake of simplicity, Fig. 4g, Fig. 4 h and Fig. $4 \mathrm{i}$ depict the results of only $\mathrm{K}$ second-stage scenarios: among the dispatching scenarios corresponding to the same unit commitment sequence, only the expected costs of the cheapest one is reported.

Table 1. Cost and optimal commitment sequence resulting from different scheduling strategies.

\begin{tabular}{|c|l|c|c|c|c|}
\hline In. SOC & & I-ARSO & M-ARSO & DO & SO \\
\hline \multirow{2}{*}{$20 \%$} & Exp. costs (\$/d) & 80.1 & 81.0 & 84.5 & 81.6 \\
\cline { 2 - 6 } & Comm. seq & 1 & 13 & - & - \\
\hline \multirow{2}{*}{$40 \%$} & Exp. costs (\$/d) & 77.5 & 80.7 & 79.4 & 77.5 \\
\cline { 2 - 6 } & Comm. seq & 1 & 95 & 95 & 1 \\
\hline \multirow{2}{*}{$60 \%$} & Exp. costs (\$/d) & 77.6 & 83.2 & 78.5 & 78.0 \\
\cline { 2 - 6 } & Comm. seq & 1 & 88 & 30 & 60 \\
\hline
\end{tabular}




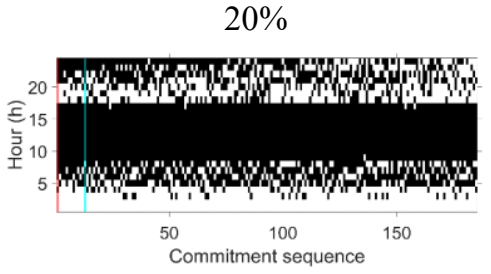

(a) Commitment sequence of the generator (white: on, black: off): in red and light blue are denoted the unit-commitment are highlighted the one of the SO procedure highlighted the one of the SO procedure highlighted the one of the SO procedure the DO technique.

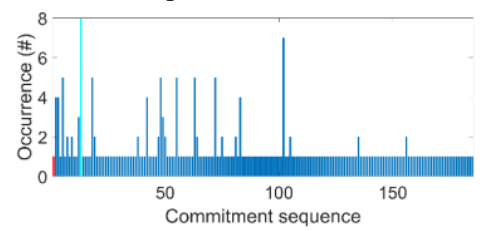

(d) Occurrence of commitment sequences: the red and light blue bars corresponds to IARSO and M-ARSO, respectively

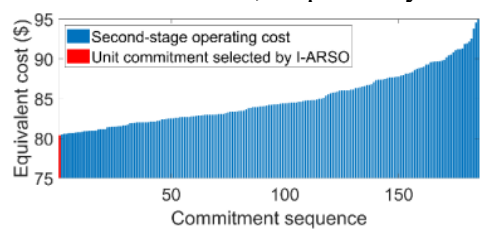

(g) Expected second-stage operating cost of different commitment sequences.
Initial SOC $(\%)$

$40 \%$

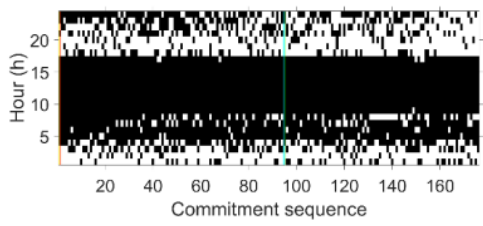

b) Commitment sequence of the generator (c) Commitment sequence of the generator (white: on, black: off): in red and light blue (white: on, black: off): in red and light blue are denoted the unit-commitment are denoted the unit-commitment equences selected by the second stage of sequences selected by the second stage of and in green the commitment sequence of and in green the commitment sequence of the DO technique.
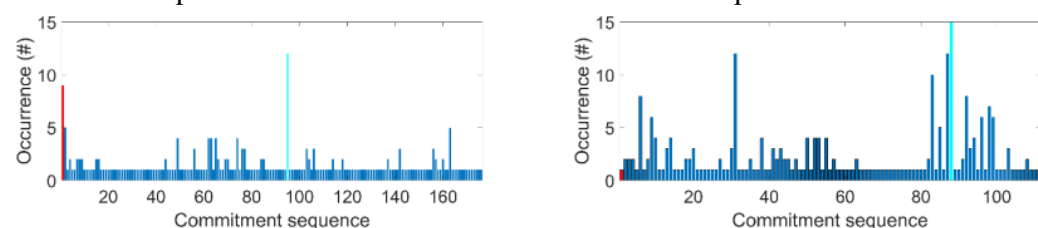

(e) Occurrence of commitment sequences: (f) Occurrence of commitment sequences: the red and light blue bars corresponds to I- the red and light blue bars corresponds to IARSO and M-ARSO, respectively

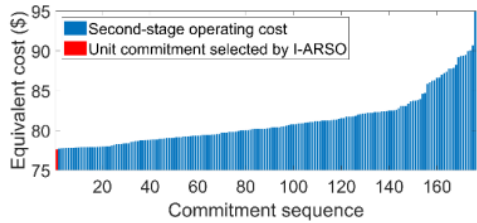
ARSO and M-ARSO, respectively

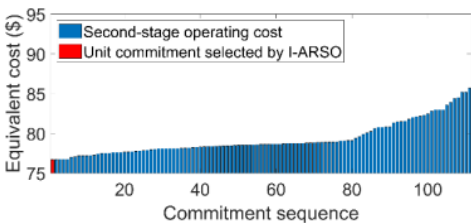

(h) Expected second-stage operating cost of (i) Expected second-stage operating cost of different commitment sequences. different commitment sequences.

Fig. 4. Analysis of the unit commitment sequences analyzed with ARSO methodologies.

According to the results shown in Tab. 1, among all tested operating strategies I-ARSO is always the cheapest one, followed by SO; DO and M-ARSO are usually much more expensive. As shown in [15], IARSO performs better than both DO and SO, due to the unit commitment of resources that is usually different from the other approaches. For example, with an initial SOC of $40 \%$, the unit commitment selected by I-ARSO is the same as the one identified by SO and consequently their final cost results are close. In other cases, the profiles are quite different, thus suggesting that the low number of scenarios of SO, limited by computational requirements, do not enable SO to really capture the stochastic nature of the load and of the renewable sources. Note that SO scenarios were limited to 6 due to the high computational requirements that can exceed 30 minutes each on average [15]; an I-ARSO optimization requires usually less than a minute to be solved.

Results shown in Fig. 4 highlight the details of IARSO and M-ARSO approaches. All commitment sequences are sorted at increasing second-stage costs, reported in Fig. 4a, Fig. 4b and Fig. 4c. This enables highlighting that the cheapest unit commitment, the first on the left, usually implies a reduced use of the generator. However, there are different unit commitments that lead to similar equivalent costs, being these images useful for the operator. However, the plot of the occurrence probability of the different commitment sequences, shown in Fig. 4d, Fig. 4e and
Fig. $4 \mathrm{f}$ is quite flat, thus suggesting that there is no clear optimal sequence. As a consequence, M-ARSO approaches can easily lead to sub-optimal solutions, while I-ARSO or SO can provide cheaper dispatches.

In Fig. 4a, Fig. 4b and Fig. 4c, as well as in Tab. 1, the commitment sequence achieved by each tested approach is highlighted. The results of I-ARSO are always the cheapest ones, i.e. the first on the left in the figures; moreover, in the case of $60 \%$ initial SOC, IARSO, M-ARSO, DO and SO select completely different operating strategies, while the same does not occur in the other cases. At $40 \%$ of initial SOC, the commitment sequence of SO equals the one of I-ARSO and, similarly, the commitment sequence of DO equals the one of M-ARSO, hence the most recurring. At $20 \%$ initial SOC, instead, no first-stage ARSO solution corresponds to the sequences selected by SO or DO.

\section{Conclusions}

In this paper we analyzed different predictive operating strategies to address uncertainties in isolated systems. Deterministic (DO) and stochastic (SO) approaches were compared to two methodologies based on the novel Aggregating-Rule-based Stochastic Optimization (ARSO), which decomposes a N-scenario stochastic problem into a number of deterministic subproblems that are later aggregated with specific rules. 
The simulation-based (I-ARSO) and mixed statisticalweighted-average (M-ARSO) rules are considered.

Results confirmed that I-ARSO approach is very promising, as it was the cheapest among all tested cases; SO follows behind I-ARSO, yet with many more computational requirements. Instead, M-ARSO methodologies are usually as expensive as DO, or slightly more. In this study, we showed that the different approaches can lead to different unit commitment sequences of the diesel generator but different optimality of results, thus the operating strategy has to be carefully chosen.

The proposed results can be the base for further studies on the novel ARSO optimization methods.

\section{References}

1. M. Franz, N. Peterschmidt, M. Rohrer, and B. Kondev, "Mini-grid Policy Toolkit," 2014.

2. REN21, Renewables 2018 Global Status Report. 2018.

3. C. D. Barley and C. B. Winn, "Optimal dispatch strategy in remote hybrid power systems," Sol. Energy, vol. 58, pp. 165-179, Oct. 1996.

4. L. Moretti, S. Polimeni, L. Meraldi, P. Raboni, S. Leva, and G. Manzolini, "Assessing the impact of a two-layer predictive dispatch algorithm on design and operation of off-grid hybrid microgrids," Renew. Energy, vol. 143, pp. 14391453, 2019.

5. D. Fioriti, D. Poli, and G. Lutzemberger, "Rolling-horizon scheduling strategies for off-grid systems : on the optimal redispatching frequency and the effects of forecasting errors," in 19th IEEE International Conference on Environment and Electrical Engineering (EEEIC), 2019.

6. D. S. Pandžić, H., Dvorkin, Y., Qiu, T., Wang, Y., and Kirschen, "Toward Cost-Efficient and Reliable Unit Commitment Under Uncertainty," IEEE Trans. Power Syst., no. 99, pp. 1-13, 2016.

7. Q. P. Zheng, J. Wang, and A. L. Liu, "Stochastic Optimization for Unit Commitment A Review," IEEE Trans. Power Syst., no. 99, pp. 112, 2015.

8. S. Y. Abujarad, M. W. Mustafa, and J. J. Jamian, "Recent approaches of unit commitment in the presence of intermittent renewable energy resources: A review," Renew. Sustain. Energy Rev., vol. 70, pp. 215-223, 2017.

9. A. J. Kleywegt, A. Shapiro, and T. Homem-deMello, "The Sample Average Approximation Method for Stochastic Discrete Optimization," SIAM J. Optim., vol. 12, no. 2, pp. 479-502, 2002.

10. H. Quan, D. Srinivasan, and A. Khosravi, "Integration of renewable generation uncertainties into stochastic unit commitment considering reserve and risk: A comparative study," Energy, vol. 103, pp. 735-745, 2016.

11. D. E. Olivares, J. D. Lara, C. A. Canizares, and M. Kazerani, "Stochastic-Predictive Energy
Management System for Isolated Microgrids," IEEE Trans. Smart Grid, vol. 6, no. 6, pp. 1-9, 2015.

12. G. Morales-España, Á. Lorca, and M. M. de Weerdt, "Robust unit commitment with dispatchable wind power," Electr. Power Syst. Res., vol. 155, pp. 58-66, 2018.

13. M. Håberg, "Fundamentals and recent developments in stochastic unit commitment," Int. J. Electr. Power Energy Syst., vol. 109, pp. 38-48, Jul. 2019.

14. Y. Wang, Y. Liu, and D. S. Kirschen, "Scenario Reduction With Submodular Optimization," IEEE Trans. Power Syst., vol. 32, no. 3, pp. 2479-2480, 2017.

15. D. Fioriti and D. Poli, "A novel stochastic method to dispatch microgrids using Monte Carlo scenarios," Electr. Power Syst. Res., vol. 175, no. October 2019, 2019.

16. E. Arriagada, E. López, M. López, R. BlascoGimenez, C. Roa, and M. Poloujadoff, "A

probabilistic economic dispatch model and methodology considering renewable energy, demand and generator uncertainties," Electr Pow Syst Res, vol. 121, pp. 325-332, 2015.

17. R. Jabbari-Sabet, S.-M. Moghaddas-Tafreshi, and S.-S. Mirhoseini, "Microgrid operation and management using probabilistic reconfiguration and unit commitment," Int. J. Electr. Power Energy Syst., vol. 75, pp. 328-336, 2016.

18. N. Nikmehr, S. Najafi-Ravadanegh, and A. Khodaei, "Probabilistic optimal scheduling of networked microgrids considering time-based demand response programs under uncertainty," Appl. Energy, vol. 198, pp. 267-279, 2017.

19. R. Siddaiah and R. P. Saini, "A review on planning, configurations, modeling and optimization techniques of hybrid renewable energy systems for off grid applications," Renew. Sustain. Energy Rev., vol. 58, pp. 376-396, 2016.

20. L. Moretti, M. Astolfi, C. Vergara, E. Macchi, J. I. Pérez-Arriaga, and G. Manzolini, "A design and dispatch optimization algorithm based on mixed integer linear programming for rural electrification," Appl. Energy, vol. 233-234, no. November 2018, pp. 1104-1121, 2019.

21. M. Giuntoli, P. Pelacchi, and D. Poli, "On the use of simplified reactive power flow equations for purposes of fast reliability assessment," IEEE EuroCon 2013, no. July, pp. 992-997, 2013. 